\title{
The Influence of the Concepts of Biophilia and Biomimicry in Contemporary Architecture
}

\author{
Pedro Ressano Garcia \\ Department of Architecture and Urban Planning, Universidade Lusófona de Humanidades e Tecnologias, Lisboa, Portugal
}

\begin{abstract}
The contemporary demand for reducing carbon emission is changing the way architects design buildings, thus influencing a wide range of new solutions. In this paper, the author presents a method that intends to contribute for the discussion of recent strategies that lower the buildings' consumption of energy. The study establishes three priority parameters to analyze the façades based on the materials, the practices and the thermal behavior. Each parameter is measured separately scaled from artificial to natural building materials, local to distant practices and insulation to inertia. The design of façades has been evolving to follow complex regulations that aim to increase the required sustainable performance of buildings. Scientific data is measurable individually by each parameter, though the cross influence between parameters raise the level of complexity. Shading systems, solar passive energy influence the measurement but the growing use of renewable energies affects the measurements of energy consumption. Each design responds differently to climatic conditions, and requires complex analyses considering the specificity of the natural environment and cultural context. The discussion makes use of scientific data that influences architectural design, the research requires a broader perception thus including cultural aspects. Recent high tech insulating systems have an effect on design solutions that characterize biophilia (human love of nature). The wisdom of traditional local solutions tested over generations holds cultural aspects of biomimicry (nature as model). The aim is to discuss whether the framework based on biophilia and biomimicry is useful for the research.
\end{abstract}

Key words: Architecture, biophilia and biomimicry, low-tech and high-tech solutions.

\section{Introduction}

Building's energy consumption is increasing at an unprecedented rhythm expanding the emission of carbon dioxide in the atmosphere. Most buildings have been adjusted to the rising standards of comfort offered by the mechanisms that artificially create a controlled environment of stable temperatures. Climate change and the consequent extreme swings in nature are adding pressure to the dominant trend.

To keep the thermal conditions pleasant inside, buildings are increasingly dependent on high-tech solutions that produce $\mathrm{CO}_{2}$. Throughout the 20th century, the dominant tendency in the western culture has been to artificially calibrate the interior environment and control the natural swings of the external environment. In the 21 st century, to adapt to climate change, architecture design is responding in a

Corresponding author: Pedro Ressano Garcia, Architect, M. Arch, Ph.D. E-mail: pedro@ressanogarcia.com. different way. The public awareness reaches a broader audience that criticizes mechanic solutions. It is expected to keep the same levels of comfort on the inside, but without damaging the environment on the outside. Depending on the technology and knowledge of the designers there is a general tendency to deal with and learn from nature in a different way.

Among the recent best practices in contemporary architecture, there are an increasing number of solutions that give priority to the existing resources and promote the use of renewable sources of energy. The production of waste shrinks and the response to nature improves. A new approach emerges and the engine of transformation is set on the dialogue with the natural environment. It offers a wide range of possibilities and influences a new philosophy related with the built environment.

The contemporary demand to reduce carbon emissions is not only a concern of developed countries where thermal studies, complex regulations and energy 
requirements are imposing new solutions for the architecture design. Developing countries are also facing the necessity to deal with reduction and the local conditions.

The present discussion focus on the need to reduce brings change. It influences the way architects design and the way they develop updated methodologies to lower energy consumption of buildings. While it opens a wide scope of approaches to architecture, the dominant factors that influence design decisions follow two dominant cultural guidelines imprinting a philosophy that covers two main areas: biophilia (love of nature) and biomimicry (nature as a model). On the opposite side, we have the biophobia (horror of nature) and bionics replacing nature by machines, e.g., air conditioning. They call for a relation of rejection or control towards nature and neglect the possibility to integrate the natural conditions of each climate in a fruitful dialogue.

This paper aims to discuss how the love for nature may influence the future of architecture. When biophilia and biomimicry are integrated in the design, they treasure a deeper understanding of the natural environment. The love of nature and the search for its balance create a deep effect in the built environment. There is a growing number of buildings aiming to reach carbon zero emission, using passive solar energy, making use of eco design and learning from geographic conditions. The contemporary paradigm mainly depends on building materials, budget and technology to deal with the local conditions; together those three influence the cultural context that covers from traditional to cutting edge technology, from high maintenance to low tech.

The solutions used in facades concentrate some of the best practices by making use of various types of insulation. Shading systems, passive energy and thermal inertia are among some of the most disseminated. They hold a central importance in the architecture intended to be discussed in this paper. The possibility to mimic nature values a sensitive relation with solutions that consume fewer energy and/or search for a symbiosis with the natural environment. Biophilic architecture shares the love for nature and the love for life in the planet. It brings back nature into the built environment in thoughtful ways, to question how architecture can be used as a tool to fight global warming?

"The one process now going on that will take millions of years to correct is the loss of genetic and species diversity by the destruction of natural habitats. This is the folly our descendants are least likely to forgive us" [1].

\section{Methodology}

The research makes use of three parameters to measure façades: practices (local or distant); materials (natural or artificial); and thermal behavior (inertia or insulation). The analysis of each case uses five grades for each topic that can swing from top to bottom. The conjugation of the parameters constructs a diagram for each situation that informs the level of proximity to the concept of biophilia or biomimicry.

Practices, depending on the region, the solutions used in the construction are classified as local or distant. Local practices respond to the local climate (hot and desert areas - adobe, in the Arctic - ice igloo, in the jungle - wood, in humid tropics - bamboo, etc.). The use of bricks widely disseminated with industrialization and cement in the 20th century is measured in its distance to the location of the production.

During the twentieth century, the use of cement and steel spread worldwide. It deeply influenced architecture production and became the most disseminated practice in modern society. The present awareness towards the amount of pollution produced by these materials, the knowledge of its implications, make them undesirable. The general efficiency when relating with the environment, energy consumption and the deterioration of the nature made them unpopular. The perception that popular modernist buildings and urban structure consume high levels of energy is raising new 
questions. This has an impact on the architecture discourse; it is becoming increasingly present and changing the way architects perceive the buildings that were references for those living in the 20th century.

As for materials, most buildings primarily make use of those that are available on site. They are classified between nature and artificial depending on the technology available (low and high). The low tech materials repeat solutions usually tested over generations; they contain effective aspects of biomimicry prior to the modern use of the machines. A particular material is considered natural when it is low tech and adapts to local conditions. It becomes traditional when copied throughout the years proving to be worth repeating. Other solutions were probably tested but did not succeed being repeated, thus proving not to adapt well to the local conditions and the environment. In the nature of materials, Hitchcock intended to present Frank Lloyd Wright from this perspective, giving evidence of his inventiveness.

Artificial materials become available due to the flow of goods in the planet, they are often high tech and not necessarily related with the local conditions. The costs per unit have a deep influence in the building industry when it comes to choose between several options. The classification of artificial is distant from traditions and local technology. The information received from traditional building technologies requires an intimate knowledge of local culture. Artificial materials can certainly reinterpret the use of the natural, however, it is the combination of both low and high tech, offering a fertile field for new design solutions.

Thermal behavior has a profound impact on the built environment. The use of insulating materials succeeds reducing the impact of temperature variations. The thermal building performance depends on the insulation and how it relates to biophilia and the love for solutions exists in the nature. It is possible to lower the consumption of energy by using design and influenced by the understanding of the phenomena within nature.
Research studies collect data which give evidence that some of the buildings with high level of thermal inertia consume less energy, keep the temperature comfortable on the inside, share the love for nature and promote a profound symbiosis between human presence and nature. Between inertia and insulation, the discussion divides conservative and progressive; the second intend to identify the implementation of best practices to highlight local solutions. Insulation, however, is more effective in conditions that require temperatures cuts.

To achieve reduction of $\mathrm{CO}_{2}$ emissions, architects' collaboration with engineers and other disciplines aims at lowering environmental impact. At present, architecture design, guided by the necessity to reduce energy consumption, is influencing how buildings are imagined and the solutions they adopt. The improvement of energy efficiency is an interdisciplinary process that is summarized in six parameters.

The aim is to discuss whether design solutions influenced by biophilia and biomimicry are useful to reduce the ecological footprint. Mimetic solutions copy nature and adapt to the specificity of the environment. The parameters used to measure are useful to understand the complexity and monitor the impact of the built environment in the natural environment. The first copies of nature were probably made intuitively, at present, parametric methods are able to turn theoretical concepts into scientific data, thus shaping the growth of this influential philosophy.

The three parameters (Fig. 1) to measure façades: practices (local or distant); materials (natural or artificial); and thermal behavior (inertia or insulation), are assembled to integrate geographic situations, budget and technology available in the surroundings to deal with the necessity to reduce carbon emissions.

Physical phenomena such as transpiration (the transition from liquid to steam cools down the temperature) and freezing (the transition from liquid to solid produces heat) are being used to regulate 
temperature. The dissemination of these and other renewable sources of energy influence building design and regulations. In this paper, the author presents a possible frame work that aims to evaluate the influence of biophilia ideas in the choice of materials, the use of passive solar energy, cross ventilation and renewable sources of energy.

\section{Diagrams}

The diagrams below (Figs. 2-12) are organized by the axes of the three parameters and construct a visual analysis of nine typologies covering different facades.

The reduction of buildings' energy consumption is depending on various parameters; insulation, solar exposure, thermal inertia, crossed ventilation, natural light, etc. As a prototype, each building operates in a unique way and the thermal performance is evaluated by rigorous data.

Individually, each parameter can be listed and measured separately but the perception of thermal comfort is a result of a complex system in which each parameter interacts and influences the other. Comfort is depending on the materials and their capacity to retain temperature and humidity, which varies from new constructions to renewable buildings and is influenced by solar orientation and the quality of air, just to list a few. To promote a systematic knowledge, quantifiable and usable scientific information, interdisciplinary teams assemble the complex relation between parameters.

To measure the consumption of energy driven by principles of sustainability, the equation deals with a wider scope of complexity. It starts during the construction period, prior to being used and when it is used, the way it performs is measured throughout the four seasons, $\mathrm{CO}_{2}$ emissions are lower when local materials are used and higher if transported from other regions. The exact same building depending on its building materials, solar orientation and shading system can be more efficient than another.

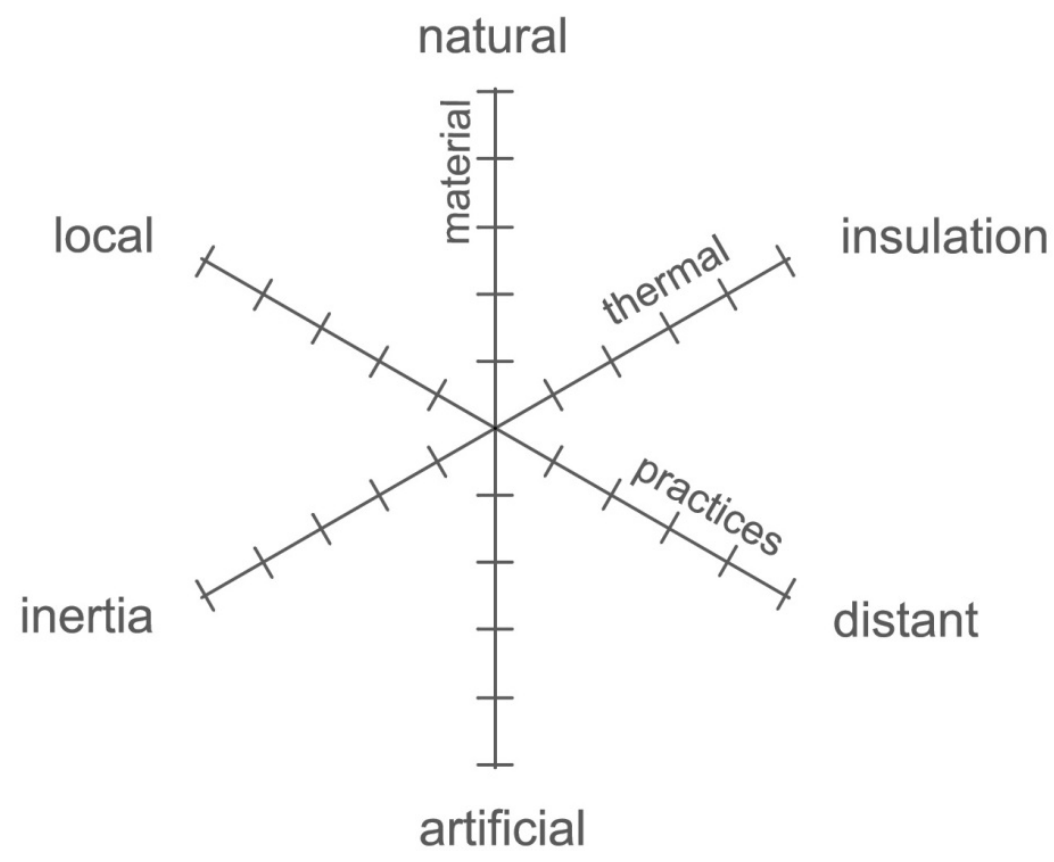

Fig. 1 Diagram combining three parameters to measure façades: practices (local or distant); materials (natural or artificial); and thermal behavior (inertia or insulation). 


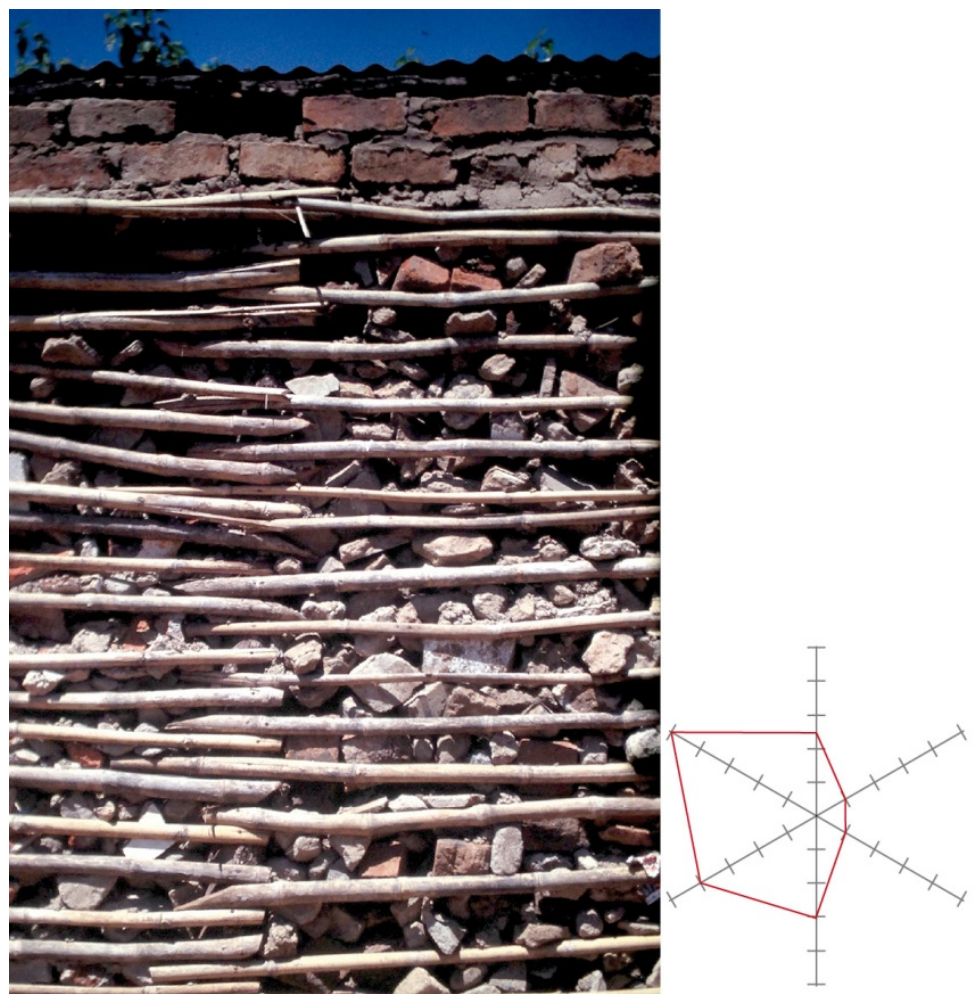

Fig. 2 Clay, stone and wood are available on site. The technology to build the frame wall has been carried out through generations of local builders (Morocco).
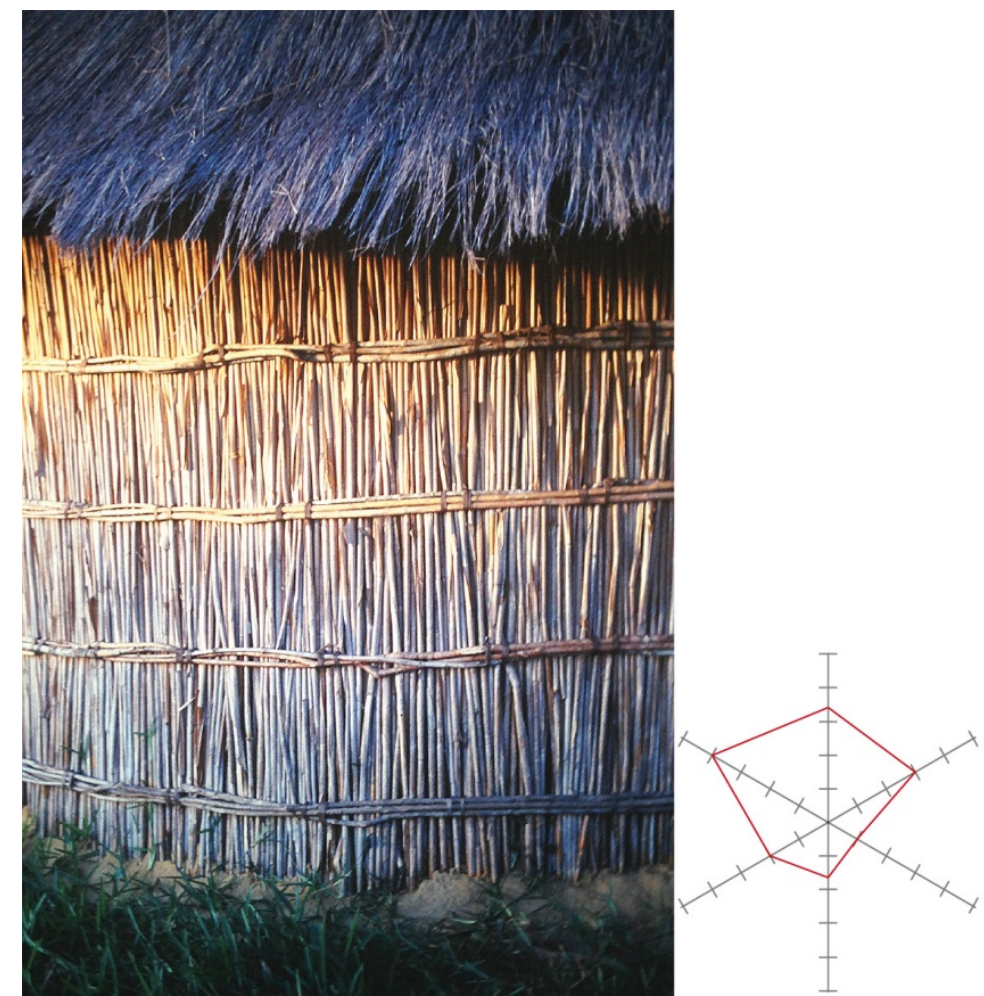

Fig. 3 External walls made with straw, abundant in the region, are widely disseminated as straw offers a good level of insulation (Inhambane, Mozambique). 

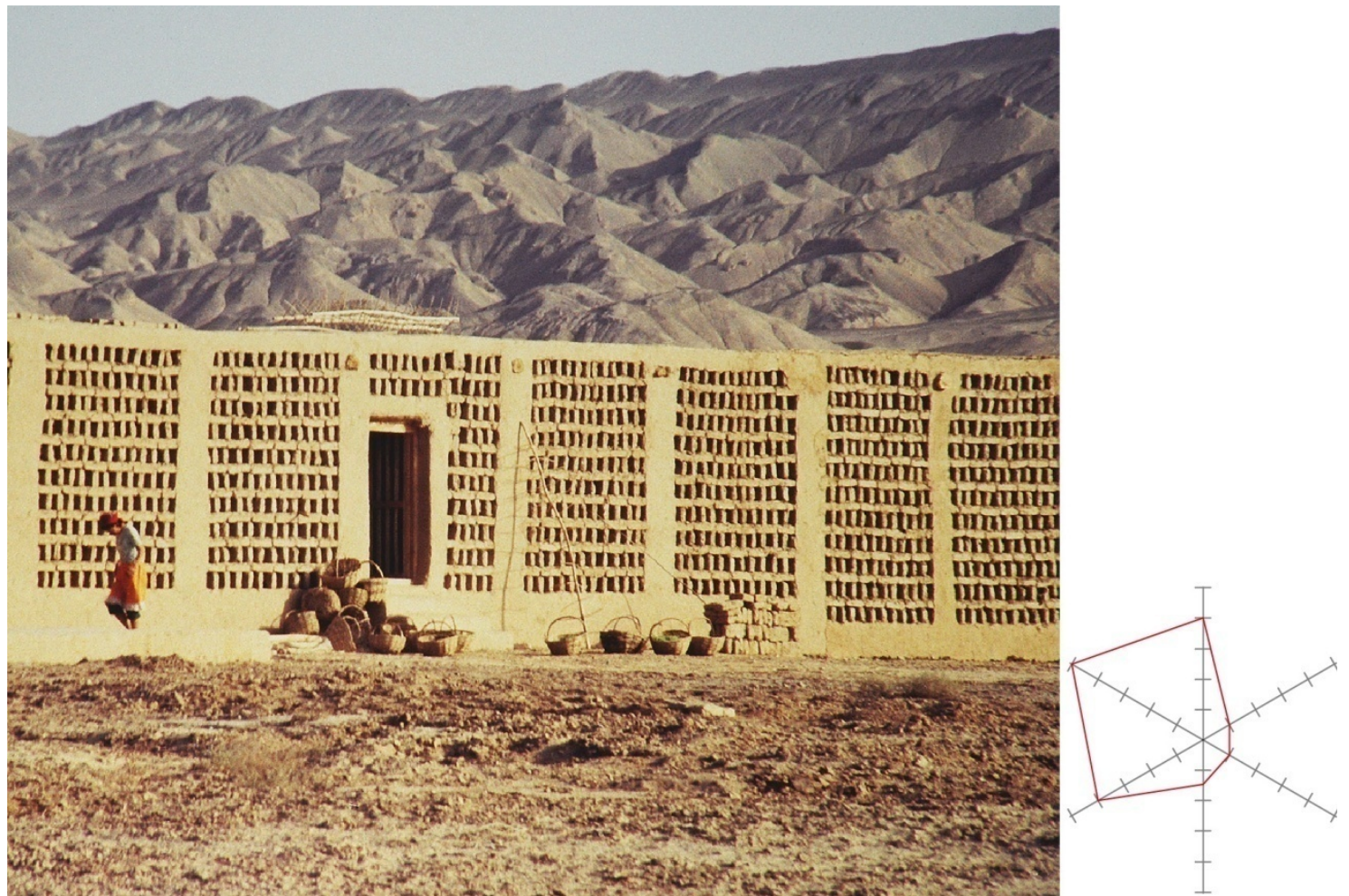

Fig. 4 The handmade bricks are built mixing, earth, clay and dry vegetation. The building system allows natural cross ventilation and moisture release during the warm period (Turpan, China).

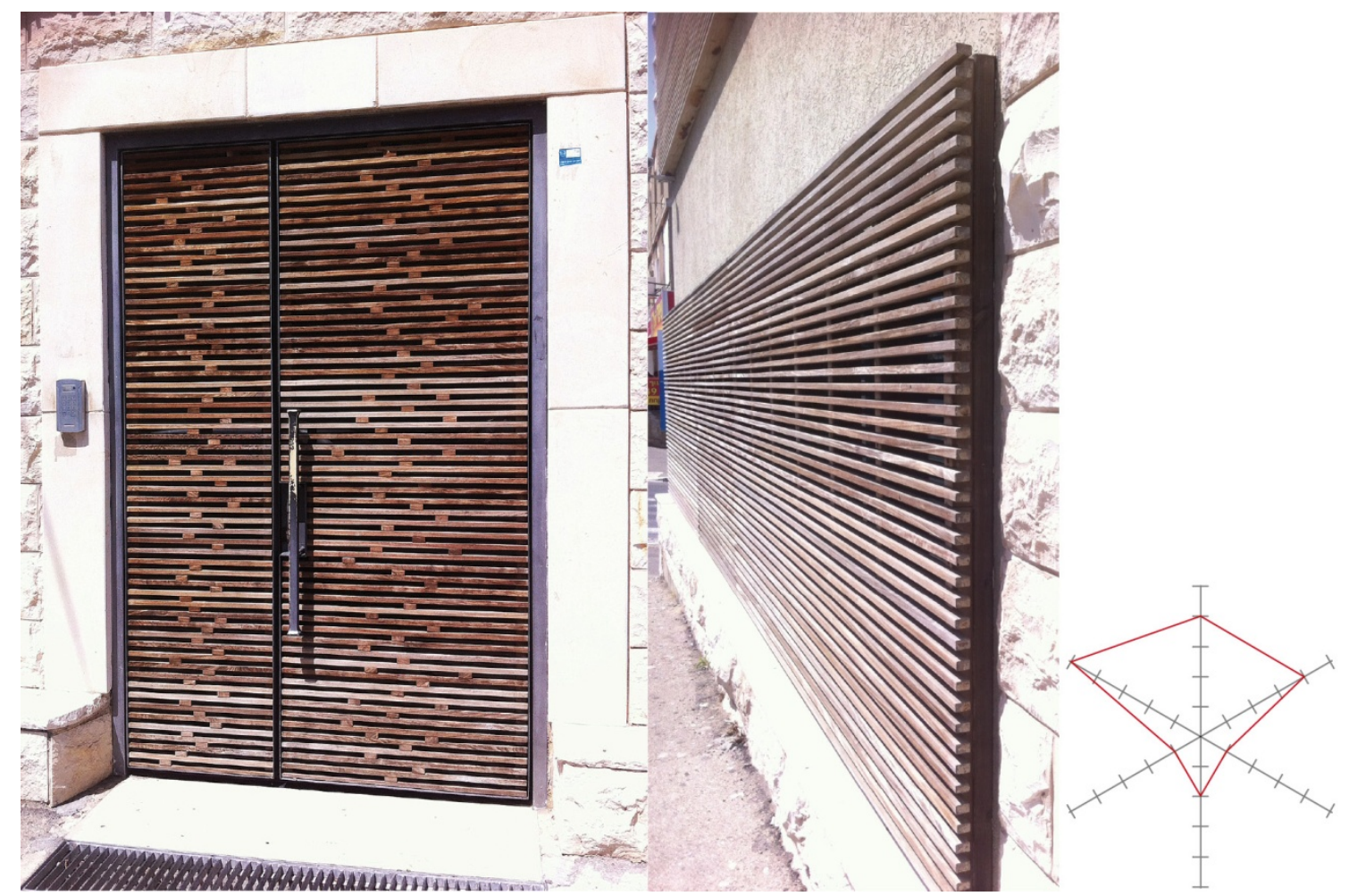

Fig. 5 Blinds, made of local wood, prevent the sun to heat up the interior and filter the natural light. A low tech solution that has been used by local carpenters in various residential building (Haifa, Israel). 

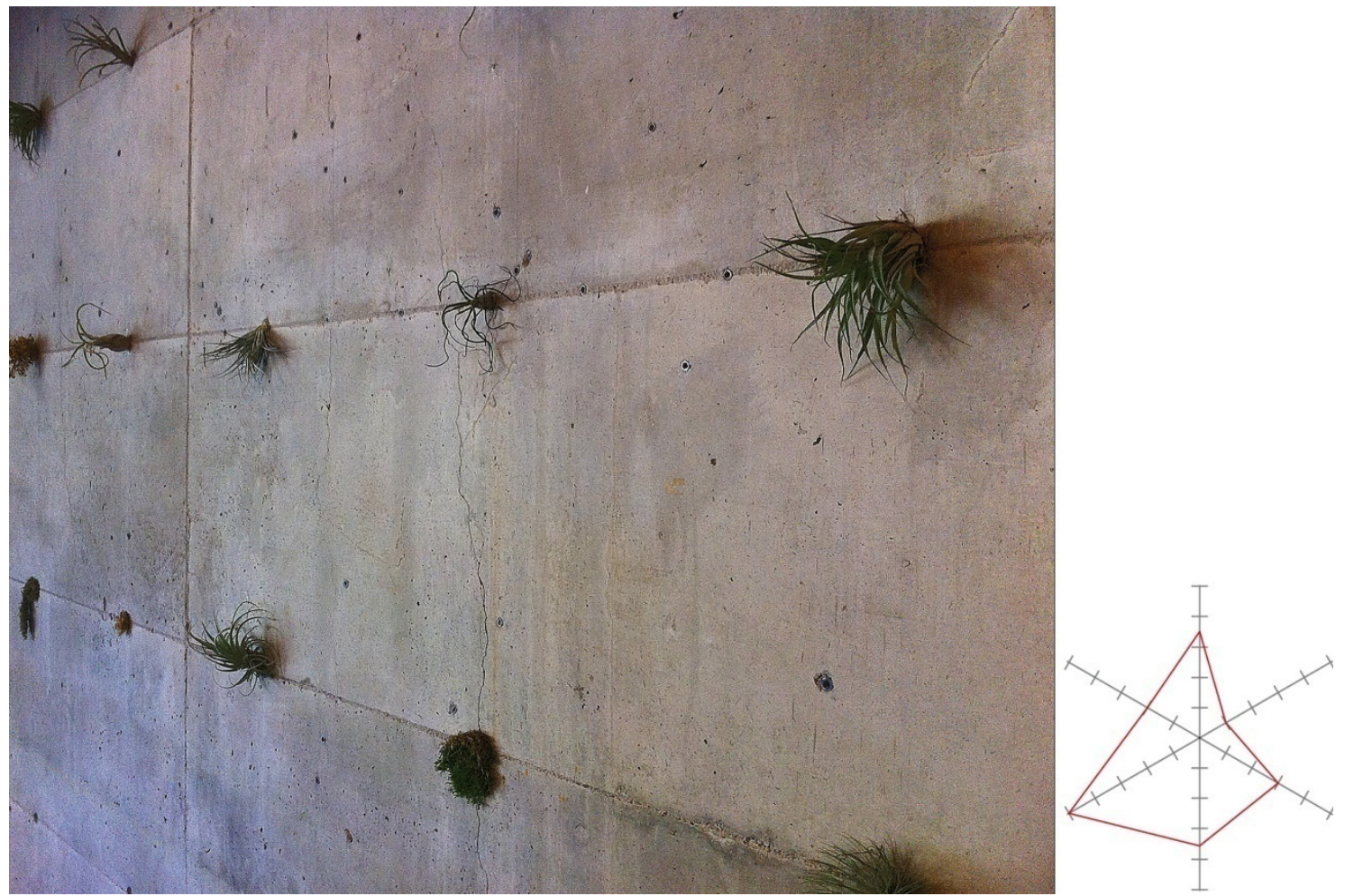

Fig. 6 Concrete wall host living vegetation on shuttering holes. The intervention, inside the Berkeley Art Museum and Pacific Film Archive by Mario Ciampi, challenges the use of concrete walls to reduce carbon emissions (Berkeley, CA, USA).
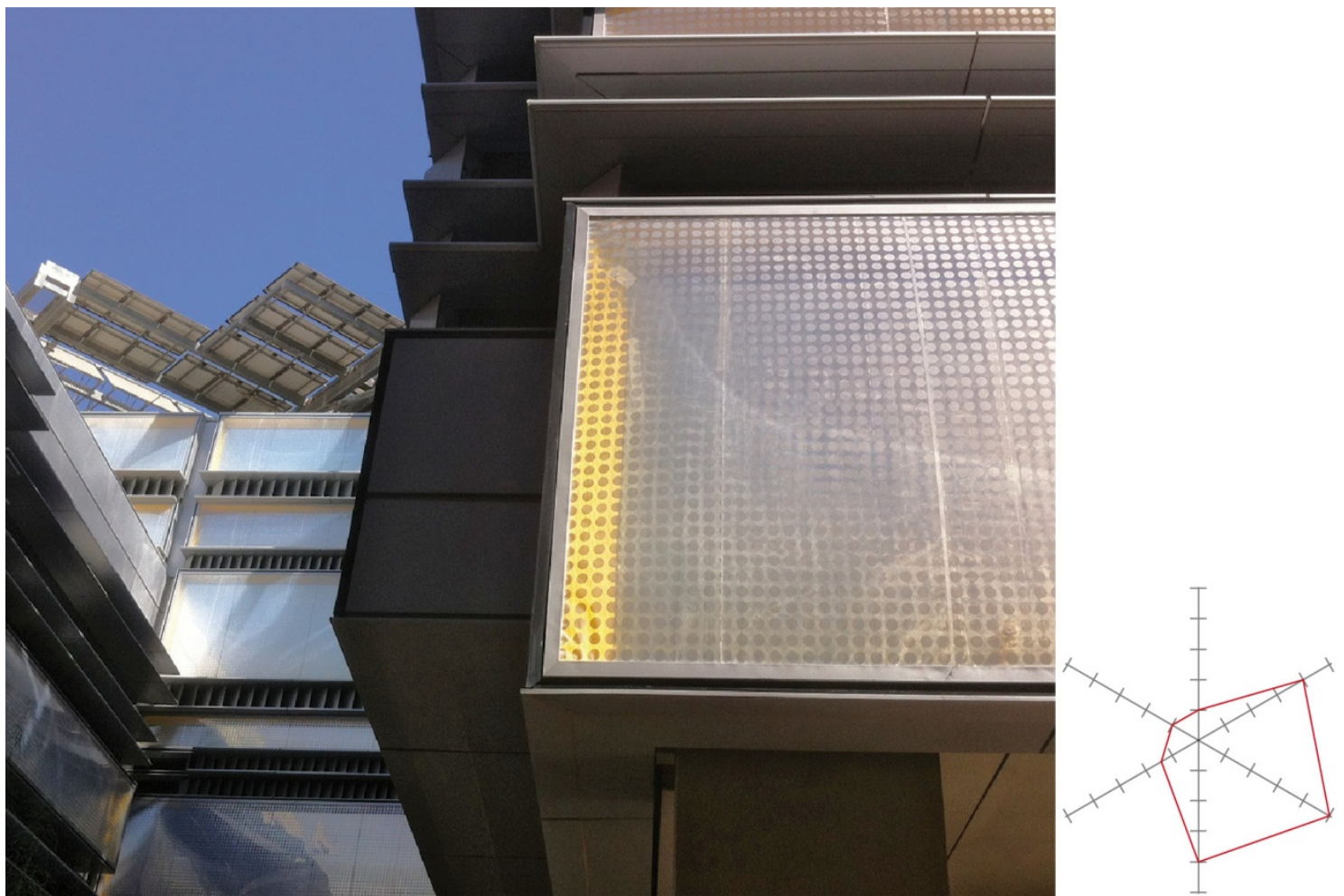

Fig. 7 The extreme thermal condition of the desert demand the use of new high tech design (air/PVC) walls built in Masdar Institute by Norman Foster (Abu Dhabi, UAE). 


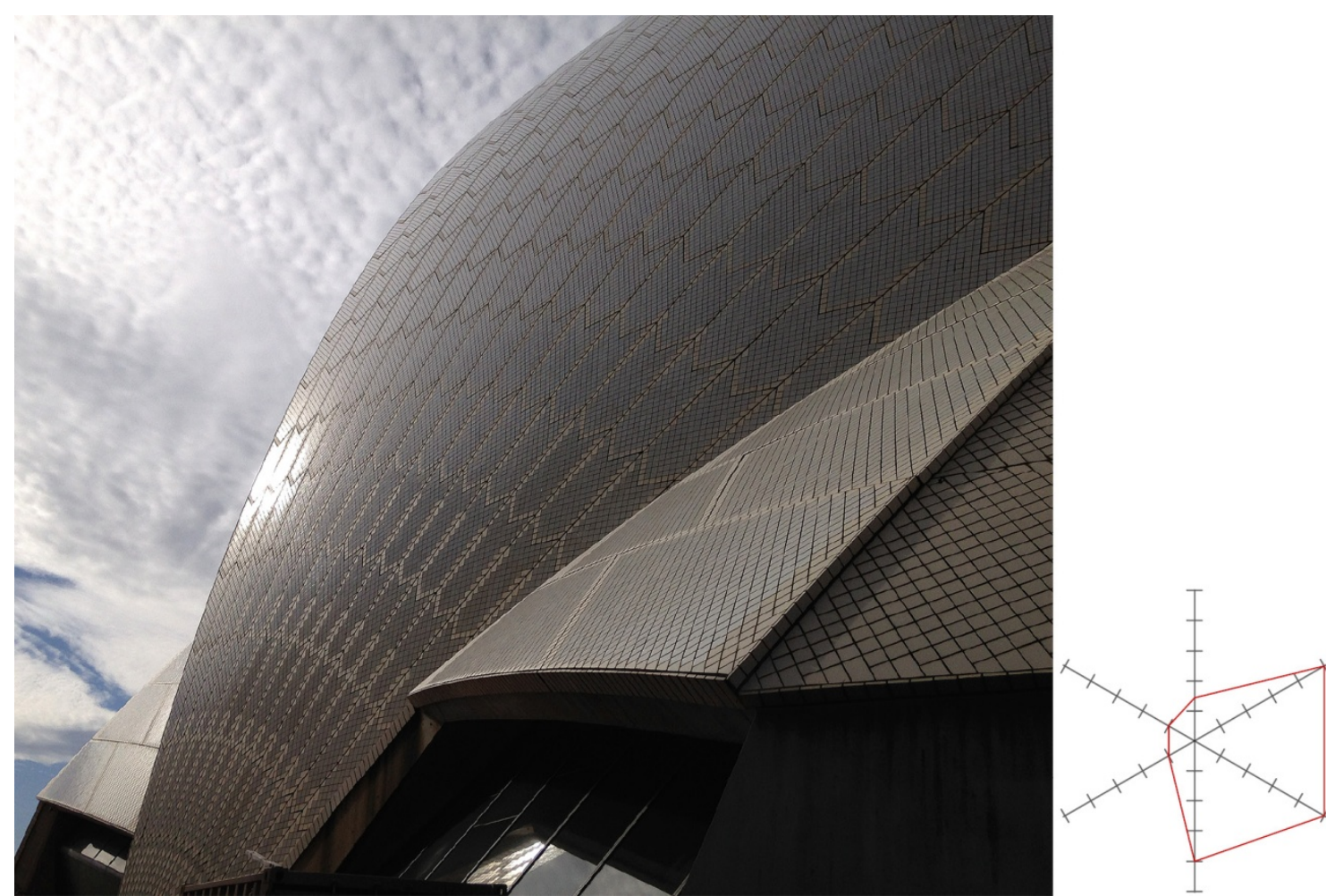

Fig. 8 The ceramic coating that overlays the concrete structure of the Opera House by Jørn Utzon contributes for the thermal behavior improvement (Sydney, Australia).
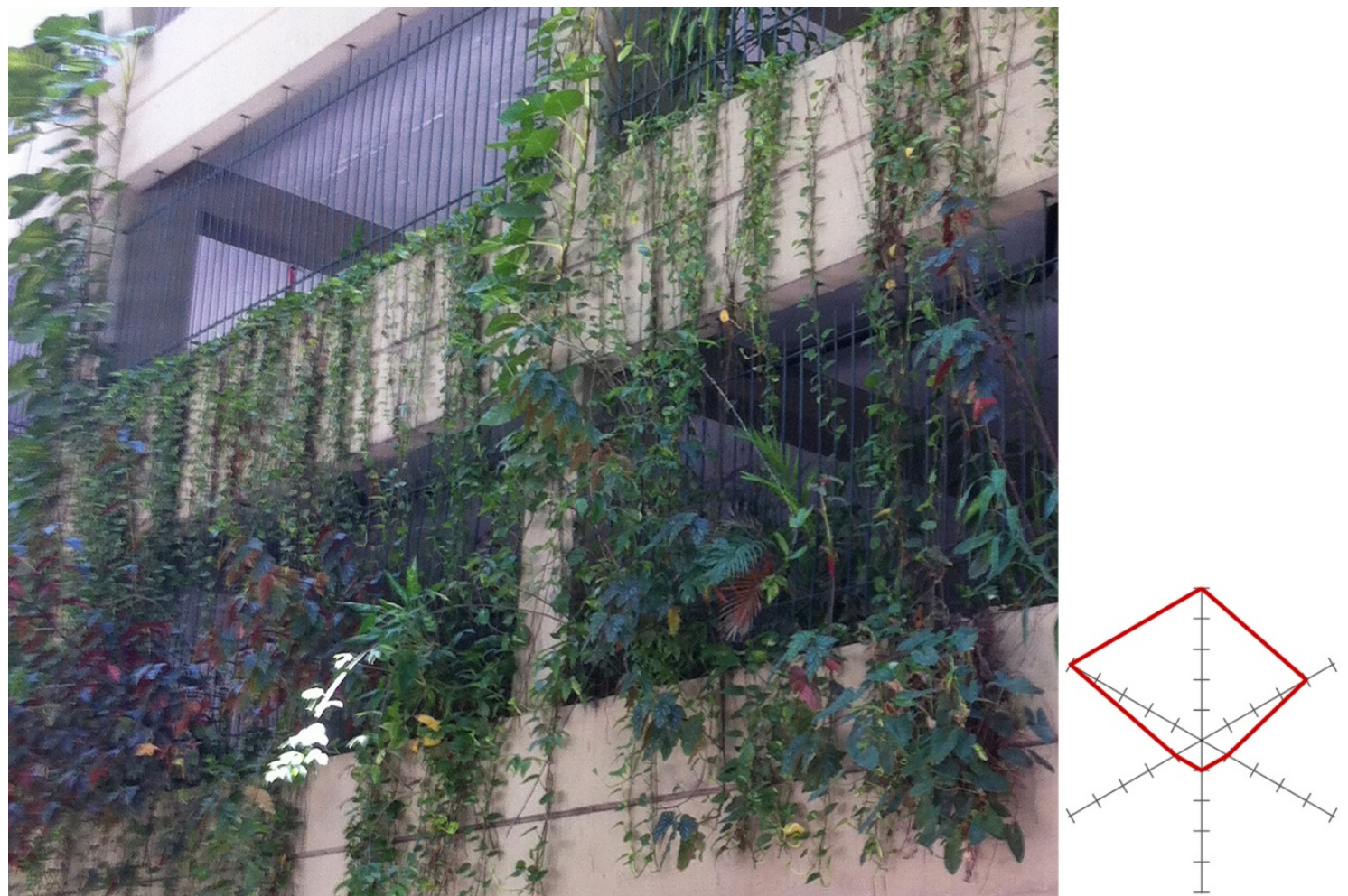

Fig. 9 Local vegetation is used to lower the temperature. Shadow, cross ventilation and moisture release succeed naturally reducing the level of high humidity and heat inside Guilherme Weinschenck building (Rio de Janeiro, Brazil). 

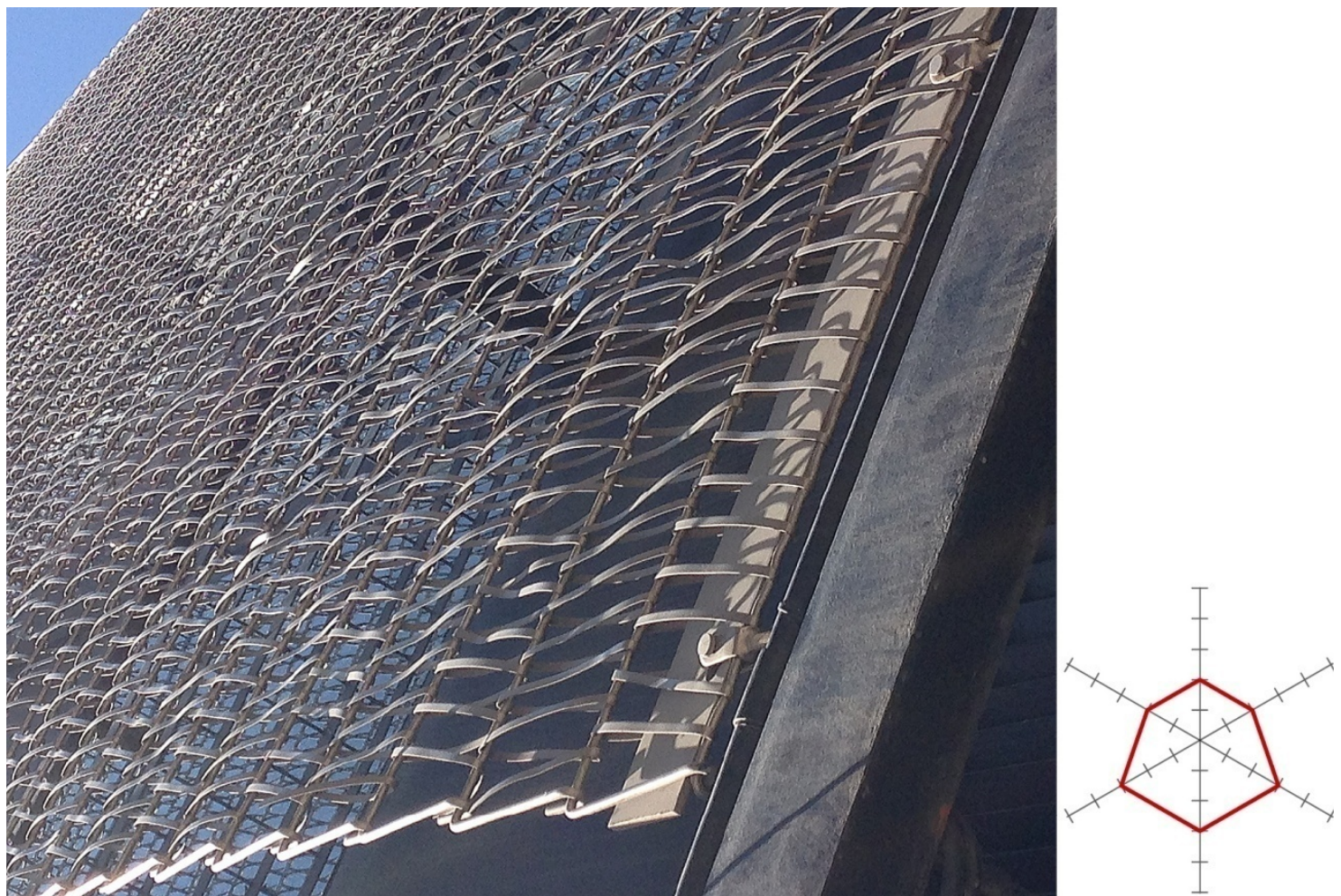

Fig. 10 The practice to cover buildings with industrial metal net produced by then regional metallurgic industry makes use of the local technology. The net covers all the Barceló Raval Hotel by CMV Architects (Barcelona, Spain).

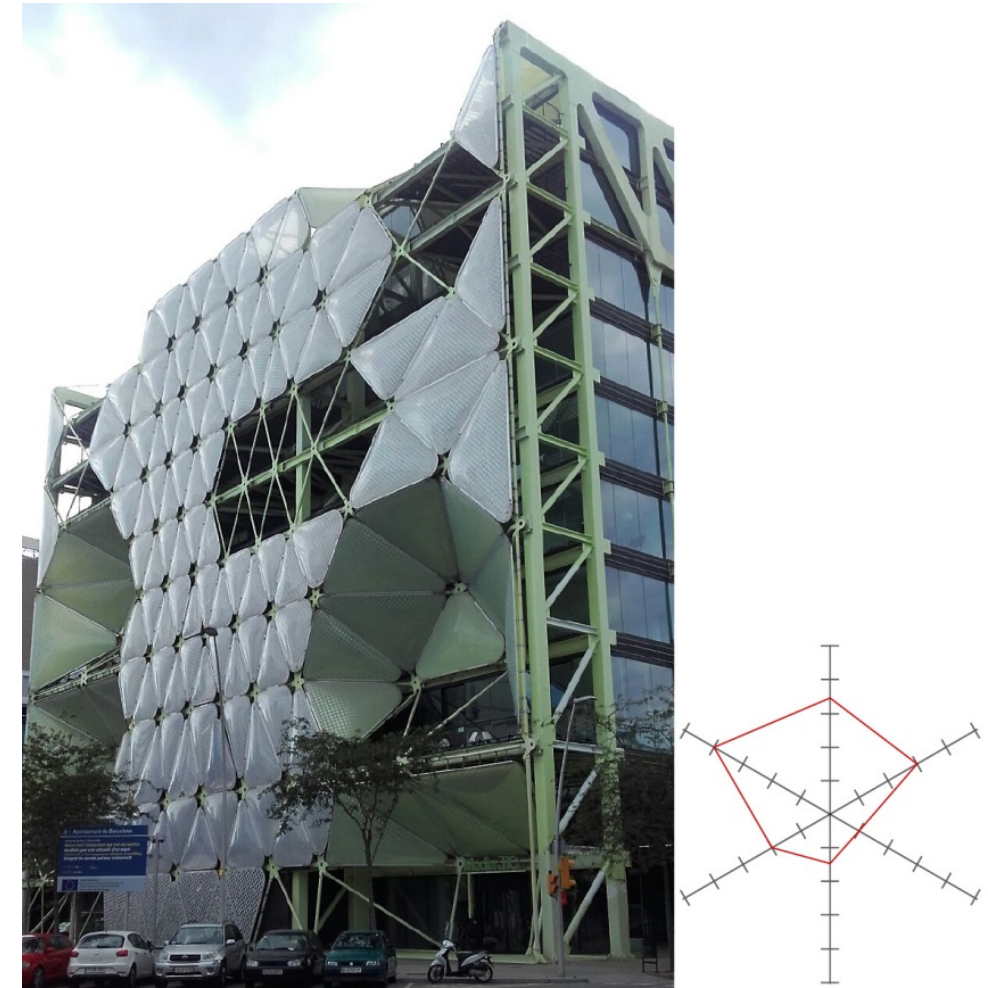

Fig. 11 Media-TIC Building by Enric Ruiz Geli makes use of high tech design (air/PVC) to lower carbon emissions and test new materials in a new neighborhood (Barcelona, Spain). 

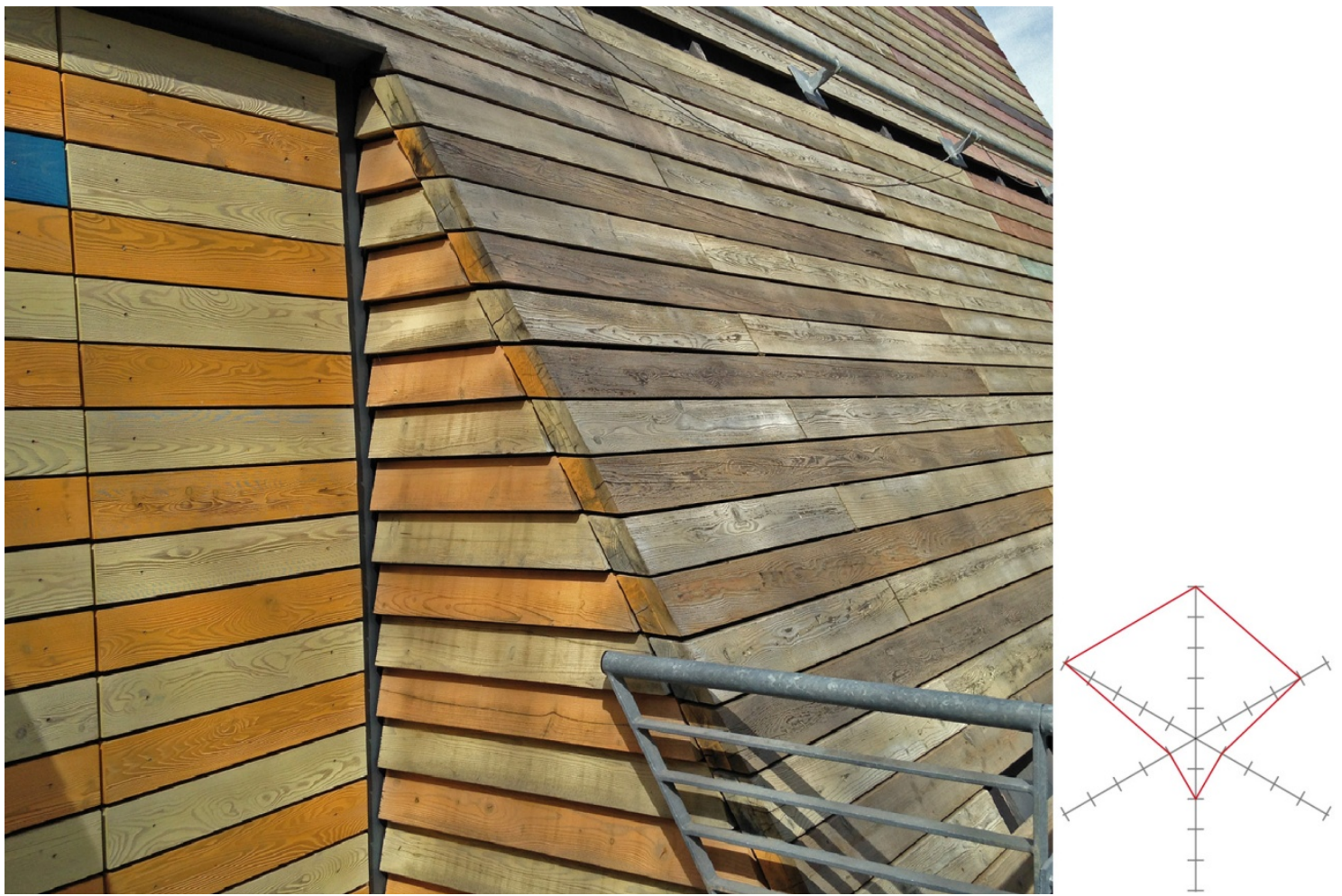

Fig. 12 Wood covers the Auditorium del Parco by Renzo Piano, to provide good insulation and respond to the urgent need followed by the 2009 earthquake (L'Aquila, Italy).

Mitigation (so limiting the emissions, low-energy development) or adaptation (resilience, living under new risks and conditions) are guidelines to evaluate the combination of parameters. Buildings are complex systems that achieve better performances when all parameters are engaged harmoniously with each other. The way parameters interact creates unpredictable results, such as in the case of Museum S. Roque in Lisbon. During the rehabilitation of the building hosting a unique collection from 1600s and 1700s, the decision to install a state of the art air conditioning intended to provide the most efficient balance of temperature and humidity on the inside of the exhibition rooms. After the installation, the records presented unpredictable picks that were harmful to the collection. After months of constant surveys, the records presented a moment of stability and optimal temperature and humidity. However, it was for a short period of time. The dates match the failure of the air conditioning mechanism, therefore engineers required more tests and constant survey. Finally, they found out that the combination of the previous materials used in the building had the capacity to provide a better condition than the mechanism. To reach the best air conditioning performance, the mechanism was not sufficient, the elaborate project did not address the complexity created by other parameters and was misused. This is often the case because each parameter is systematically evaluated independently from other parameters; it is quantifiable and usable for further scientific information however, away from a holistic approach.

"While defining an equilibrium between the welcome solar gain in winter and defense against the undesired solar gain in summer, any kind of shading devices, operable and permanently installed need to be implemented into the design process" [2].

\section{Low Tech and High Tech-Biophilia}

In "14 Patterns of Biophilic Design" [3], Bill Browning articulates the relationships between nature, human biology and the design of the built environment 
so that we may experience the human benefits of biophilia in our design applications.

The contemporary best practices of architecture projects are those investing in the dialogue with the natural environment and the possibilities to naturally control thermal comfort. They explore concepts based on knowledge of local culture, combine to learn from nature while implementing strategies that make use of new technologies. The combination of concepts, biophilia and biomimetics, enables a working methodology based on three principles:

(1) use of natural or artificial materials;

(2) practical application of local or distant solutions;

(3) thermal control, inertia and isolation.

Global solutions are quickly spread through digital information and specialized media. The present technology allows architects to exchange information with other professionals anywhere in the world. Shading systems have been developing the capacity to reduce energy consumption inside the building [4]. Insulation is one of the key factors, however building sustainability requests the capacity to simultaneously insulate and keep the temperature. Thermal inertia, lightening comfort, etc., are also influencing the performance of the energy consumption of the building.

Crossing data to provide the most efficient building design is possible through the use of computer programs that succeed evaluating and monitoring the performance throughout the day and across the seasons of the year.

Intelligent shading systems, the use of stainless steel or light metal structures, the possibility to measure efficient solar passive energy are among some of the current possibilities offered to architects at unprecedented level. High tech when mixed with secular knowledge hold by low tech is holding the most innovative solutions of contemporary architecture.

Construction methods that are driven by principles of sustainability and water supply with recycling capacity are also included in the project. The search for efficient building design makes use of cutting edge technologies that provide accurate information regarding thermal and lighting comfort, thus influencing the use of materials and design strategies in search of optimal behavior. The state of the art software provides accurate data for the best solutions to be integrated in design.

"The term 'mashrabiya' literally means 'place used to store drinks' and it is a small projection in a dim light, that served for support of small jars that needed to stay cool; (...) is a window element that automatically activates a convective cycle that moves air masses from the zone of high pressure to that of low pressure. (...) ensure ventilation of the room through humidity and impurities filtering system, (...)." [5].

\section{Biomimicry Solutions-Traditional and New}

Through the perception of biomimicry that is the imitation of nature, the author records solutions that have been tested for centuries in traditional houses still existing in the region. To reduce carbon emissions is developing energy efficiency strategies where different methodologies adopted focus on the behavior of the materials used and the thermal control solutions.

Local solutions repeated over centuries are good and proved to be efficient. Some experimental solutions were also implemented, but since they were not good, they were not repeated or carried out to the next generation. Traditional building design holds such knowledge. Solutions are tested over time and only the best are kept and copied.

The possibilities offered by the use of updated software in the design process allow architects to test buildings thermal behavior throughout the year before they are built. While insulation provided by the recent materials is of growing efficiency, thermal comfort is also highly dependent on thermal inertia best offered by materials such as earth, adobe and stone. Earth buildings [6], for instance, have been associated with low tech communities and are generally related to low 
scale constructions.

In the Mediterranean, the integration of clay in the inner surfaces of the building has been used for centuries. The material holds an extraordinary capacity to keep temperature and humidity on the inside providing high levels of comfort for the user. Usually, interiors were well ventilated and protected from bright light common in the warm season. The transpiration of walls lowers the temperature because the transition from liquid to steam cools down the temperature - it is a natural phenomenon.

In the cold regions, designers are introducing ice heating solutions, when water changes from liquid to solid, it produces heat that is used to regulate temperature without energy consumption. The transition from $0^{\circ}$ to $2^{\circ}$ is made by solar energy, thus disseminating a zero carbon emission solution based on a natural phenomenon.

In the cold season, buildings that did not perform well as they did not take advantage of solar passive energy to warm up the interiors and insulation was not used regularly, are capable of improving comfort through biomimicry without damaging nature.

Most construction methodologies followed common criteria for centuries, which results to the materials available at hand. Clay, stone, sand and earth are used in many existing buildings, holding a good thermal inertia. At present, rehabilitations are associated with materials available, such as plaster, stucco, concrete, steel and cement that do not have the same characteristics. Insulations materials produced by high tech industries are widely disseminated, although the employment of natural cork, which holds an extraordinary insulation capacity [7], has been used by most constructions in the cork tree region. Insulation regulations improve the performance of the building but alone are not capable to evaluate the influence of biomimicry ideas in contemporary building design.

\section{Conclusions}

To evaluate the façade's relation to the concepts of biophilia and biomimicry, the research tested a methodology based on three parameters; practices (local or distant); materials (natural or artificial); and thermal behavior (inertia or insulation). Each parameter is graded according to its specific criteria that take in consideration the relation to nature and the ability to imitate it. The combination of parameters was useful to evaluate each case and construct a diagram for each façade. Each diagram informs the relation to the concept of biophilia or biomimicry thus contributing to decrease carbon's emissions.

One of the main arguments of this paper is that innovative solutions emerge from the mixture of high tech and low tech. High tech is often depending on elaborate software and the new possibilities they offer to design solutions. Low tech is spread out in traditional building design holding solutions that have been tested over centuries. In general, there are more biophilia (love of nature) cases that make use of high tech strategies and low tech solutions tend to relate to biomimicry (nature as a model). The distinction of biophilia and biomimicry is inappropriate because they search for the similar aims to reduce carbon emission and adapt to climate change.

The solutions used in facades make use of insulation, materials and practices to establish a possible base for discussion and creation of a body of knowledge of best practices on the subject. They do not offer an exact framework for designers. Passive energy and thermal inertia are strategies well disseminated among facades that mimic nature. Some of the cases reveal that it is the conjugation of the parameters that enhance the proximity to the love for nature and the love for a symbiosis with the natural environment.

The research presented intended to question a possible methodology to evaluate whether architecture could be a tool to fight global warming by bringing back nature into the built environment.

The influence of the concepts holds a central 
importance in the architecture intended to be discussed in this paper. The possibility to mimic nature values a sensitive relation with solutions that consume less energy and/or search for a symbiosis with the natural environment. Low tech buildings made with local materials that have been tested over centuries are most likely to integrate high technologies and contemporary materials that are being spread worldwide. Global and local are promoting environmental quality project based on traces of ancient urban settlements in the southern region and policy of the $3 \mathrm{Rs}$ : reduce, re-use and recycle.

Each system responds differently to climate conditions and requires careful analysis within the context. Best practices sought to integrate tradition and modernity, highlighting interventions that avoid conflicts between materials. Biophilic architecture shares the love for nature and the love for life in the planet; therefore, it is most likely to influence the contemporary architecture.

\section{References}

[1] Wilson, E. O. 1984. Biophilia. Cambridge: Harvard University Press.

[2] Heine, U. 2010. "Design and Validation Premises and Concept for the Design of an Affordable Low Energy Architecture." Sustainable Architecture and Urban Development IV.

[3] Browning, B. 2014. 14 Patterns of Biophilic Design: Improving Health and Well-being in the Built Environment. New York, NY: Terrapin Bright Green.

[4] Kellert, S. R. 2008. "Dimensions, Elements, and Attributes of Biophilic Design.” In Biophilic Design: The Theory, Science and Practice of Bringing Buildings to Life, edited by Kellert, S. R., Heerwagen, J., and Mador, M. Hoboken, N.J.: John Wiley \& Sons.

[5] Ficarelli, L. 2010. "Passive Cooling in Traditional Construction: Case of Domestic Architecture in Egypt." Sustainable Architecture and Urban Development I: 231-45.

[6] Baglioni, E. 2010. “Terra: Material Sustentável.” In El Diseño de la Arquitectura de Tierra. Salto, Montevideo: Uruguai, 179-83. (in Spanish)

[7] Oliveira, M. A., and Oliveira, L. 2000. Cork. Portugal: Amorim. 


\section{Annex: Complementary Bibliography}

[1] Bencheikh, H. 2015. "The Effect of Wind Velocity and Night Natural Ventilation on the Inside Air Temperature in Passive Cooling Ventilation in Arid Zones.” In Renewable Energy in the Service of Mankind, edited by Sayigh, A. Vol. I. Switzerland: Springer International Publishing, 423-32.

[2] Birkeland, J. 2002. Design for Sustainability: A Sourcebook of Integrated, Eco-logical Solutions. London, UK: Earthscan Publications.

[3] Birkeland, J. 2007. "Ecological Waste: Rethinking the Nature of Waste.” BEDP Environment Design Guide 1: 1-9.

[4] Demirbilek, N., and Ecevit, A. 2001. "Solar Houses." Enerji Dunyasi, DunyaEnerji Konseyi Turk Milli Komitesi Bulteni (World Energy Council Turkish National Committee Bulletin), 42-7.

[5] Elkadi, H. 2006. Cultures of Glass Architecture. Hampshire, UK: Ashgate.

[6] Ficarelli, L. 2010. "Passive Cooling in Traditional Construction: Case of Domestic Architecture in Egypt." Sustainable Architecture and Urban Development I: 231-45.

[7] Rashid, R., and Ahmad, M. H. 2009. Green Influence on the Building's Level of Comfort and Wellbeing of Urban Residents and Sustainability. Tripoli, Libya: Sustainable Architecture and Urban Development, CSAAR,.

[8] Salim, F. N. 2011. Cultural Sustainability and Changing Worldview: Dilemmas of Architecture and the Built Form, The Constructed Environment. Champaign, IL: Common Ground Publishing.

[9] Snell, C., and Callahan, T. 2009. Building Green: A Complete How-to Guide to Alternative Building Methods-Earth Plaster, Straw Bale, Cordwood, Cob, Living Roofs. N.Y., Asheville, United States: Lark Books.

[10] Tang, G. 2003. The Lobstermata-Hybrid between the Lobster and the Stomata-A Conceptual Architectural Analogy Drawing Similarities between the Architecture and the Environmental Mechanistic Control in the Natural World. London, England: Bartlett School of Architecture Publications.

[11] Wake, S. J., and Eames, C. 2013. "Developing an 'Ecology of Learning' within a School Sustainability Co-design Project with Children in New Zealand." Local Environment 18: 305-22. 